\title{
Performance of Patients with Relapsing-Remitting Multiple Sclerosis on the Wechsler Memory Scale-Fourth Edition (WMS-IV): Preliminary Clinical Findings
}

\author{
Samuel Thomas Gontkovsky ${ }^{1}$, Joseph John Ryan², Laura Glass Umfleet ${ }^{3}$ \\ ${ }^{1}$ Twin Valley Behavioral Healthcare, Department of Psychology, Columbus, Ohio, The United States \\ ${ }^{2}$ University of Central Missouri, School of Nutrition, Kinesiology, and Psychological Science, Warrensburg, Missouri, The United States \\ ${ }^{3}$ Medical College of Wisconsin, Department of Neurology, Milwaukee, Wisconsin, The United States
}

Email address:

Samuel.Gontkovsky@mha.ohio.gov (S. T. Gontkovsky)

To cite this article:

Samuel Thomas Gontkovsky, Joseph John Ryan, Laura Glass Umfleet. Performance of Patients with Relapsing-Remitting Multiple Sclerosis on the Wechsler Memory Scale-Fourth Edition (WMS-IV): Preliminary Clinical Findings. Clinical Neurology and Neuroscience.

Vol. 5, No. 2, 2021, pp. 13-17. doi: 10.11648/j.cnn.20210502.12

Received: March 9, 2021; Accepted: March 29, 2021; Published: April 26, 2021

\begin{abstract}
Historically, the Wechsler Memory Scales have been one of the most commonly used measures of memory in clinical neuropsychological evaluations. There are limited published reports, however, analyzing the performance of patients with multiple sclerosis (MS) on the Fourth Edition of the test. The aim of this investigation was to describe the utility of the Wechsler Memory Scale-Fourth Edition (WMS-IV) in detecting and characterizing the memory deficits associated with relapsing-remitting MS. Thirty-four outpatients with clinically definite relapsing-remitting MS were administered the WMS-IV and Wechsler Adult Intelligence Scale-Fourth Edition (WAIS-IV). Means for age, education, and duration of MS diagnosis were $43.15,14.88$, and 8.41 years, respectively. Index scores did not differ significantly across the five WMS-IV domains. Comparison of actual WMS-IV indexes with those predicted by the WAIS-IV General Ability Index revealed that in every instance predicted index means were significantly higher than actual index means. Only $6(17.6 \%)$ of 34 patients had all five actual and predicted index scores at comparable levels; whereas, $28(82.4 \%)$ had $\geq 1$ actual indexes significantly below the predicted level. Contrary to prior research using earlier versions of these measures, more patients demonstrated relatively reduced performances on the WMS-IV Visual Working Memory Index than on the WAIS-IV Processing Speed Index. Results support the use of the WMS-IV in evaluating learning and memory in individuals with MS. Findings also challenge the notion that the information processing deficits in MS are more reflective of reduced processing speed than impaired working memory.
\end{abstract}

Keywords: Assessment, Learning, Neuropsychology, Processing Speed, Working Memory

\section{Introduction}

Performance of Patients with Relapsing-Remitting Multiple Sclerosis on the Wechsler Memory Scale-Fourth Edition (WMS-IV): Preliminary Clinical Findings

Cognitive dysfunction, including memory impairment, has long been established as a common behavioral deficit in patients with multiple sclerosis (MS) [1-3], with impairment prevalence estimates generally ranging from 40 to $65 \%$ [4-6]. Such deficits more recently have come to be viewed as one of the most disabling symptoms of the disease [7], and detrimental consequences of cognitive impairment may include premature retirement, increased psychopathology and sexual dysfunction, reduced social engagement, and overall poorer quality of life [8]. Moreover, it has been reported that memory disorders often are the earliest and most common adaptive problems associated with MS, regardless of disease type or duration [9].

Within the clinical milieu, the California Verbal Learning Test-Second Edition (CVLT-II) [10] is the instrument of choice for the assessment of patients with MS-related memory deficits [11]. The Wechsler Memory Scales, however, have been among the most widely used memory batteries for over 65 years and in a relatively recent survey of neuropsychological practitioners [12], the Wechsler Memory Scale-Revised (WMS-R) [13] and Wechsler Memory Scale- 
Third Edition (WMS-III) [14] were ranked higher than the CVLT-II in frequency of test usage. It is reasonable to assume that the WMS-IV will be ranked higher in test usage frequency than the CVLT-II if and when the next survey of neuropsychological practitioners is published.

Multiple studies have investigated the clinical utility of the WMS-R and WMS-III in characterizing the memory functioning of patients with MS [15-17]. In general, findings have revealed mildly reduced performances, overall, but with considerable variability across individuals. Information processing deficiencies also are a common form of cognitive impairment among individuals with MS, and several researchers have investigated the relative contributions of processing speed abilities and working memory abilities to this deficit. Relevant to the current study are the findings of DeLuca, Chelune, Tulsky, Lengenfelder, and Chiaravalloti [18] in which the Processing Speed Index (PSI) of the Wechsler Adult Intelligence Scale-Third Edition (WAIS-III) [19] was reported to contribute more to deficient information processing in patients with MS than the Working Memory Index of the WMS-III.

The Fourth Edition of the Wechsler Memory Scale (WMSIV) [20] was intended to supersede its predecessors and, if past clinical practice patterns of test usage are maintained in the future, will soon or may already be the most widely utilized memory battery within the clinical setting. There exist few published reports, however, describing the clinical use of the WMS-IV in patients with MS. The present investigation therefore was undertaken to provide information on the WMS-IV performance of patients afflicted with the relapsing-remitting type of MS. The study tested the three primary hypotheses that (1) patients with MS will demonstrate significant variability across the five index scores/memory domains of the WMS-IV, (2) actual index scores of patients with MS will be significantly below ability-based predicted index scores at both the group and individual levels, and (3) primary information processing deficits in patients with MS will reflect speed of processing as measured by the PSI of the Wechsler Adult Intelligence Scale-Fourth Edition (WAIS-IV) [21] as opposed to working memory as measured by the Visual Working Memory Index (VWMI) of the WMS-IV.

\section{Method}

Participants were 34 outpatients ( 8 males, 26 females) with relapsing-remitting MS [22] seen at a medical center in the midwestern United States for routine clinical neuropsychological evaluation. All patients were diagnosed by a board-certified neurologist specializing in MS and were referred for formal testing to assess potential neurocognitive impairment. Patients had no medically documented deficits in vision or upper extremity motor functioning, and all participants demonstrated adequate visual acuity to read without any specialized aids other than prescription lenses as well as sufficient upper extremity/hand motor function to write without any difficulty. Further, no patients were actively applying for disability status at the time of their evaluation. As part of a more comprehensive battery of measures, each participant was administered the WAIS-IV and WMS-IV by a psychology technician under the supervision of a senior clinical neuropsychologist. No patients were experiencing an exacerbation of illness at the time of testing; whereas, the majority of participants were taking prescribed medications. Participants consisted of 33 Caucasians and 1 African American, and $94 \%$ of the sample was right-hand dominant. Means for age, education, and duration of MS diagnosis were 43.15 ( $S D=9.19), 14.88$ $(S D=2.27)$, and $8.41(S D=7.18)$ years, respectively. Means for the WAIS-IV PSI, WAIS-IV General Ability Index (GAI), WAIS-IV Full Scale IQ, and Expanded Disability Status Scale (EDSS) [23] were 91.24 $(S D=15.65), 95.09$ $(S D=13.91), 93.00 \quad(S D=14.96)$, and $3.73 \quad(S D=1.44)$, respectively. Twenty-three individuals had EDSS scores $\leq 4.5$ and 11 had scores of $\geq 5.0$.

Pearson correlation coefficients were calculated to examine the relationships among WMS-IV index scores and age, education, duration of MS diagnosis, and EDSS scores. Using table B. 1 (pp. 200-201) provided in the $W M S-I V$ Technical and Interpretative Manual [24], predicted index scores were determined based on each patient's GAI score. Predicted WMS-IV index scores were then compared to actual index scores. Finally, patient scores on the WAIS-IV PSI and WMS-IV VWMI were compared mainly by analyzing the number of individuals with scores $\geq 1$ standard deviation but $<2$ standard deviations below the means and $\geq$ 2 standard deviations below the means. This retrospective study utilizing archival clinical data received full institutional review board approval and was conducted in accordance with the Helsinki declaration and the ethical principles of the American Psychological Association [25].

\section{Results}

No significant associations were found among WMS-IV index scores and patient demographic or disease-related variables, and only the relationship between the VWMI and education showed a trend towards significance $(p=.10)$. Onesample $t$-tests revealed that across all WMS-IV indexes, the obtained scores of patients with MS were significantly below the standard mean of 100 , with $t$ values ranging from -4.29 to -5.58 , all $p \mathrm{~s}<.0005$.

In order to determine if there was meaningful variability across the five index scores, a one-way repeated measures ANOVA was run with indexes as the repeated measure. The index scores did not differ significantly across the five WMSIV domains, $F(4,132)=1.16, p=.331$. Table 1 provides means and standard deviations for GAI-predicted indexes compared to actual indexes. In all instances, mean predicted indexes were significantly higher than were actual mean indexes based on paired samples $t$-tests (all $p \mathrm{~s}<.0005$ ). Comparisons for individual participants yielded significantly lower actual versus predicted index scores for $55.9 \%$ on the Auditory Memory Index (AMI), $70.6 \%$ on the Visual Memory Index 
(VMI), $52.9 \%$ on the VWMI, $64.7 \%$ on the Immediate Memory Index, and $61.8 \%$ on the Delayed Memory Index. Of the 34 participants, six $(17.6 \%)$ had all five actual and predicted index scores at comparable levels; whereas, 28
(82.4\%) had $\geq 1$ actual index significantly below the predicted level. The distribution of impaired index scores is presented in Table 2.

Table 1. Means and standard deviations for GAI predicted indexes compared to actual indexes.

\begin{tabular}{|c|c|c|c|c|c|c|}
\hline \multirow{2}{*}{ Variable } & \multicolumn{2}{|c|}{ Predicted WMS-IV Index } & \multicolumn{2}{|c|}{ Actual WMS-IV Index } & \multirow{2}{*}{$R$} & \multirow{2}{*}{ Mean Diff. } \\
\hline & $M$ & $S D$ & $M$ & $S D$ & & \\
\hline AMI & 97.44 & 7.49 & 87.94 & 14.65 & .47 & $9.50 *$ \\
\hline VMI & 97.21 & 8.30 & 87.94 & 14.94 & .59 & $9.27 *$ \\
\hline VWMI & 96.79 & 9.27 & 88.91 & 15.09 & .75 & $7.88 *$ \\
\hline IMI & 96.79 & 9.27 & 87.12 & 15.49 & .63 & $9.68 *$ \\
\hline DMI & 97.12 & 8.16 & 85.56 & 15.10 & .49 & $11.56^{*}$ \\
\hline
\end{tabular}

$* p<.0005$

Table 2. Frequency of impaired index scores for MS patients.

\begin{tabular}{llll}
\hline Impaired Indexes & Frequency & Percentage & Cumulative \\
\hline 0 & 6 & 17.6 & 17.6 \\
1 & 2 & 5.9 & 23.5 \\
2 & 4 & 11.8 & 35.3 \\
3 & 4 & 11.8 & 47.1 \\
4 & 7 & 20.6 & 67.7 \\
5 & 11 & 32.3 & 100.0 \\
\hline
\end{tabular}

As expected, the scores of $91.24(S D=15.65)$ on the WAIS-IV PSI and $88.91(S D=15.09)$ on the WMS-IV VWMI were positively and significantly correlated with one another, $r=.63, p<.0005$, although the overall means scores obtained by patients on these indexes were not significantly different. Nevertheless, by comparison, 6 and 11 individual patients scored $\geq 1$ standard deviation but $<2$ standard deviations below the means on the PSI and VWMI, respectively. Similarly, 4 and 5 individual patients scored $\geq 2$ standard deviations below the means on the PSI and VWMI, respectively. Taken together, $29 \%$ of patients demonstrated reduced performances on the PSI; whereas, $47 \%$ of patients demonstrated reduced performances on the VWMI.

\section{Discussion}

The first hypothesis of this investigation was not supported, as patients with MS demonstrated similar mean scores across the five WMS-IV indexes. Failure to find significant index variability differs from research using the WMS-III. On the 1997 scale, 25 patients had Visual Immediate (81.5) and Visual Delayed (82.2) indexes that were substantially smaller than their mean Auditory Immediate (97.7) and Auditory Delayed (92.8) indexes. It was noted that the WMS-IV sample had similar mean index scores, yet when cases were examined individually, $71 \%$ earned a VMI significantly below their ability-based predicted level, but only $56 \%$ were below the ability-based predictions for the AMI [26]. Differences between the current and previous study may reflect the small samples that were studied (25 versus 34 ) or perhaps differences in sample composition in terms of disease subtype. We assessed patients with the relapsingremitting subtype of MS; the WMS-III investigation did not specify disease subtype. This is potentially important since disease subtypes may differ in both degree and pattern of cognitive dysfunction [27]. Finally, results may differ across studies because of structural and content changes introduced by the 2008 scale revision.

The second hypothesis was supported in that all five mean GAI-predicted index scores were significantly larger than the actual mean index scores, suggesting at least mild impairment of learning and memory in patients with MS as measured by the WMS-IV. When individual cases were considered, the majority of participants demonstrated one or more domains in which the obtained index was significantly below the ability-based estimated index. If cognitive dysfunction is defined as $\geq 2$ indexes significantly below the GAI-predicted indexes, $75.5 \%$ of the sample was impaired. Using a more conservative cut-off of $\geq 3$ actual indexes significantly below the ability-based index estimates identified $64.7 \%$ of participants as impaired. It is noted that these findings may underestimate the true magnitude of memory impairment in the present sample. The possibility exists that the GAIs used to predict WMS-IV indexes were themselves compromised by the cognitive sequelae of MS. If the GAI is compromised by the disease process, then the ability-based predicted memory index value will be reduced, as well. In a recent investigation, substantial numbers of patients with relapsing-remitting MS demonstrated possible intellectual deterioration on the WAIS-IV [28]. In a sample of 40 patients, $55.0 \%$ (22/40) of the Verbal Comprehension and $67.5 \%(27 / 40)$ the Perceptual Reasoning indexes, when combined comprise the GAI, were significantly lower than premorbid demographically based estimates. On the Full Scale IQ, approximately $78 \%$ of participants earned scores that were suggestive of cognitive decline.

The third and final hypothesis not only was unsupported by the results but was contradictory to expectation based on prior research. A larger percentage of patients with MS demonstrated reduced performances on the WMS-IV VWMI than the WAIS-IV PSI, which therefore would suggest indirectly that the information processing deficit associated with MS may be more reflective of reduced visual working memory than reduced processing speed. This finding must be interpreted with caution, however, given that the relative contributions of the PSI and VWMI to information processing could not be analyzed directly since no independent measure of this ability was administered in this 
study. As with hypothesis 1 , this unexpected finding may be the result of a change in the 2008 scale revision, which in this case involved a change from a more general Working Memory Index to an emphasis on visual stimuli.

These preliminary results have important implications for practitioners using the WMS-IV in the evaluation of patients with MS. The primary limitations of this investigation are the small sample size and the absence of comparison groups, such as a sample of demographically-matched healthy controls and/or patients with other medical disorders with similar neuropathological and behavioral manifestations. In addition, effort testing was not included as part of the battery for patients in this sample, which may have influenced results Indeed, recent research suggests that a proportion of patients with MS, in particular those applying for disability status or having significant depressive symptomatology, may perform below expectation on performance validity testing [29]. Future research in this area should address these limitations and consider direct comparison of the WMS-IV with other measures of memory, in particular the CVLT-II. In addition, in order to more precisely examine the speed versus accuracy confound among individuals with MS, future research paralleling that of Genova, Lengenfelder, Chiaravalloti, Moore, and DeLuca [30] is critical for assessing the relative contributions of processing speed abilities and working memory abilities, as measured by the Fourth Editions of the WAIS and WMS, respectively, to more pure tests of information processing, such as the Keeping Track Task utilized by Genova et al., which was lacking in this investigation.

\section{Conclusion}

It may be concluded from this study that the WMS-IV is an effective measure for use in the neuropsychological evaluation of individuals with MS. The test is sensitive to the memory disturbances associated with the disorder. The WMS-IV also may be useful in characterizing the information processing deficits of MS, especially when used in conjunction with the WAIS-IV.

\section{Conflict of Interest Statement}

The authors declare that they have no competing interests.

\section{References}

[1] Rao, S. M., Grafman, J., DiGiulio, D., Mittenberg, W., Bernardin, L., Leo, G. J., Unverzagt, F. (1993). Memory dysfunction in multiple sclerosis: Its relation to working memory, semantic encoding, and implicit learning. Neuropsychology, 7, 364-374.

[2] Rao, S. M., Leo, G. J., Bernardin, L., \& Unverzagt, F. (1991). Cognitive dysfunction in multiple sclerosis. I. Frequency, patterns, and prediction. Neurology, 41, 685-691.
[3] Rao, S. M., Leo, G. L., \& St. Aubin-Faubert, P. (1989). On the nature and memory disturbance in multiple sclerosis. Journal of Clinical and Experimental Neuropsychology, 11, 699-712.

[4] Amato, M. P., Portaccio, E., Goretti, B., Zipoli, V., Hakiki, B., Giannini, M., Razzolini, L. (2010). Cognitive impairment in early stages of multiple sclerosis. Neurological Sciences, 31 (Suppl. 2), S211-S214.

[5] Amato, M. P., Zipoli, V., \& Portaccio, E. (2006). Multiple sclerosis-related cognitive changes: A review of crosssectional and longitudinal studies. Journal of the Neurological Sciences, 245, 41-46.

[6] Rao, S. M. (1995). Neuropsychology of multiple sclerosis. Current Opinion in Neurology, 8, 216-220.

[7] Benedict, R. H. B., DeLuca, J., Enzinger, C., Geurts, J. J. G., Krupp, L. B., \& Rao, S. M. (2017). Neuropsychology of multiple sclerosis: Looking back and moving forward. Journal of the International Neuropsychological Society, 23, 832-842.

[8] Beatty, W. W. (2008). Assessment for rehabilitation of multiple sclerosis. In S. T. Gontkovsky \& C. J. Golden (Eds.), Neuropsychology within the inpatient rehabilitation environment (pp. 99-131). Hauppauge, NY: Nova Science.

[9] Meshkova, K. S., \& Damulin, I. V. (2011). Memory disorders in multiple sclerosis. Neuroscience and Behavioral Physiology, 41, 871-877.

[10] Delis, D. C., Kramer, J. H., Kaplan, E., \& Ober, B. A. (2000). California Verbal Learning Test (2 ${ }^{\text {nd }}$ ed.). San Antonio, TX: The Psychological Corporation.

[11] Benedict, R. H. B., Fischer, J. S., Archibald, C. J., Arnett, P. A., Beatty, W. W., Bobholz, J., Munschauer, F. (2002). Minimal neuropsychological assessment of MS patients: A consensus approach. The Clinical Neuropsychologist, 16, 381-397.

[12] Rabin, L. A., Barr, W. B., \& Burton, L. A. (2005). Assessment practices of clinical neuropsychologists in the United States and Canada: A survey of INS, NAN, and APA Division 40 members. Archives of Clinical Neuropsychology, 20, 33-65.

[13] Wechsler, D. (1987). Wechsler Memory Scale-Revised. San Antonio, TX: The Psychological Corporation.

[14] Wechsler, D. (1997). Wechsler Memory Scale-Third Edition. San Antonio, TX: The Psychological Corporation.

[15] Clemmons, D. C., Fraser, R. T., Rosenbaum, G., Getter, A., \& Johnson, E. (2004). An abbreviated neuropsychological battery in multiple sclerosis vocational rehabilitation: Findings and implications. Rehabilitation Psychology, 49, 100-105.

[16] Drew, M., Tippett, L. J., Starkey, N. J., \& Isler, R. B. (2008). Executive dysfunction and cognitive impairment in a large community-based sample with multiple sclerosis from New Zealand: A descriptive study. Archives of Clinical Neuropsychology, 23, 1-19.

[17] Fischer, J. S. (1988). Using the Wechsler Memory ScaleRevised to detect and characterize the memory deficits of multiple sclerosis. Clinical Neuropsychologist, 2, 149-172.

[18] DeLuca, J., Chelune, G. J., Tulsky, D. S., Lengenfelder, J., \& Chiaravalloti, N. D. (2004). Is speed of processing or working memory the primary information processing deficit in multiple sclerosis? Journal of Clinical and Experimental Neuropsychology, 26, 550-562. 
[19] Wechsler, D. (1997). WAIS-III administration and scoring manual. San Antonio, TX: The Psychological Corporation.

[20] Wechsler, D. (2009). Wechsler Memory Scale-Fourth Edition. San Antonio, TX: The Psychological Corporation.

[21] Wechsler, D. (2008). Wechsler Adult Intelligence Scale-Fourth Edition. San Antonio, TX: The Psychological Corporation.

[22] Polman, C. H., Reingold, S. C., Banwell, B., Clanet, M., Cohen, J. A., Filippi, M., Wolinsky, J. S. (2011). Diagnostic criteria for multiple sclerosis: 2010 revisions to the McDonald Criteria. Annals of Neurology, 69, 292-302.

[23] Kurtzke, J. F. (1983). Rating neurologic impairment in multiple sclerosis: An expanded disability status scale (EDSS). Neurology, 33, 1444-1452.

[24] Wechsler, D. (2009). WMS-IV: Technical and interpretive manual. San Antonio, TX: Pearson.

[25] American Psychological Association. (2002). Ethical principles of psychologists and code of conduct. American Psychologist, 57, 1060-1073.
[26] The Psychological Corporation. (2002). WAIS-III/WMS-III: Technical manual (Updated). San Antonio, TX: Author.

[27] Zakzanis, K. K. (2000). Distinct neurocognitive profiles in multiple sclerosis subtypes. Archives of Clinical Neuropsychology, 15, 115-136.

[28] Ryan, J. J., Gontkovsky, S. T., Kreiner, D. S., \& Tree, H. A. (2012). Wechsler Adult Intelligence Scale-Fourth Edition performance in relapsing-remitting multiple sclerosis. Journal of Clinical and Experimental Neuropsychology, 34, 571-579.

[29] Galioto, R., Dhima, K., Berenholz, O., \& Busch, R. (2020). Performance validity testing in multiple sclerosis. Journal of the International Neuropsychological Society, 1-8. doi: $10.1017 / \mathrm{S} 1355617720000466$.

[30] Genova, H. M., Lengenfelder, J., Chiaravalloti, N. D., Moore, N. B., \& DeLuca, J. (2012). Processing speed versus working memory: Contributions to an information processing task in multiple sclerosis. Applied Neuropsychology: Adult, 19, 132140. 